\title{
Using the Years-of-Healthy-Life Measure to Calculate QALYs
}

Peter A. Muennig, MD, MPH, Marthe R. Gold, MD, MPH

Background: The quality-adjusted life year (QALY) is an attractive outcome measure because it captures both health-related quality of life (HRQL) and life expectancy in a single metric. We present a method for calculating QALYs that is simple, utilizes data that are free of charge, and may improve consistency in burden-of-disease investigations.

Methods: For purposes of illustration, we calculated the burden of disease due to stroke using two abridged life tables, each adjusted for HRQL. The first life table was generated using all-cause mortality and morbidity data (a reference cohort) and the second was generated using all diseases except stroke (a stroke-free cohort). The difference in total QALYs and in quality-adjusted life expectancy (QALE) was determined by subtraction.

Results: Approximately 61,328 (95\% CI=60,272, 62,383) QALYs were lost to stroke in the life-table cohort. Stroke is responsible for a decrement of 0.03 years of life expectancy and 0.61 years of QALE in the United States.

Conclusions: The "years of health life"measure affords a rapid, inexpensive, and sensitive means for estimating the burden of disease for local health priorities and may assist research efforts in including QALYs as an outcome measure.

Medical Subject Headings (MeSH): cost-benefit analysis, health status, health status indicators, population characteristics, quality-adjusted life years (Am J Prev Med 2001;20(1):35-39) (C) 2001 American Journal of Preventive Medicine

\section{Introduction}

$\mathrm{B}$ ecause the quality-adjusted life year (QALY) can capture both health-related quality of life (HRQL) and life expectancy in a single metric, it is an attractive outcome measure for cost-effectiveness analyses and burden-of-disease calculations. ${ }^{1}$ However, since calculating QALYs may be complicated, expensive, and time consuming, ${ }^{2}$ some investigators may be reluctant to include them in their research. This may be especially true when limited funding is available with which to conduct a study or when rapid burden-ofdisease assessments are needed. Moreover, burden-ofdisease estimates may not be comparable when different data sources are used and may not fully capture comorbid illness or disability when data are limited. Techniques that permit ready calculation of QALYs using comprehensive data are therefore helpful to investigations intended to support public health agency

From the Robert J. Milano Graduate School, New School University (Muennig); and Department of Community Health and Social Medicine, City University of New York Medical School (Gold), New York, New York

Address correspondence and reprint requests to: Peter A. Muennig, MD, MPH, 66 5th Ave., 9th Floor, New York, NY 10011. E-mail: muennigp@newschool.edu. resource allocation decisions under conditions of uncertainty.

The National Health Interview Survey (NHIS) is an annual survey of the U.S. population containing selfreported illness, role function, and perceived health status. A QALY-compatible research tool, the "years of healthy life" (YHL) measure has been used to generate a simple "off-the-shelf" list of HRQL scores for the calculation of quality-adjusted life expectancy (QALE) in the United States using mortality data and data from the NHIS. ${ }^{3-5}$ The National Center for Health Statistics (NCHS) has created a matrix of 30 possible HRQL values by combining five levels of self-reported health and six levels of role function and linking these states to a multiattribute utility model, described in detail by Erickson et al. ${ }^{4}$ The HRQL values for the different cells have been linked to self-reported conditions. ${ }^{5}$ Though these data may be limited by the use of only two dimensions of HRQL and self-report bias, it is possible to adjust these parameters for demographic factors and to thereby capture the average HRQL of specific populations..$^{5,6}$

Given that the NHIS data may be combined with mortality data that are simple to access and use, developing a means of using the YHL measure to calculate both QALYs and QALE may shorten the time to com- 


\begin{tabular}{|c|c|c|c|c|c|c|c|c|c|c|}
\hline $\begin{array}{l}\text { Age } \\
\text { interval }\end{array}$ & \begin{tabular}{l}
\multicolumn{1}{c}{1} \\
Probability \\
of death in \\
interval
\end{tabular} & \begin{tabular}{l}
\multicolumn{1}{c}{2} \\
Number \\
beginning \\
interval
\end{tabular} & \begin{tabular}{l}
\multicolumn{1}{c}{3} \\
Number \\
dying in \\
interval
\end{tabular} & $\begin{array}{l}4 \\
\begin{array}{l}\text { Person-years } \\
\text { in interval }\end{array}\end{array}$ & \begin{tabular}{l}
\multicolumn{1}{c}{5} \\
Cumulative \\
person-years
\end{tabular} & $\begin{array}{l}\quad 6 \\
\quad 6 \\
\text { Life } \\
\text { expectancy }\end{array}$ & $\mathbf{H R Q L}^{a}$ & QALYs & \begin{tabular}{l}
\multicolumn{1}{c}{9} \\
Cumulative \\
QALYs \\
\end{tabular} & QALE \\
\hline$<45$ & 0.050 & 100,000 & 5,004 & $4,405,191$ & $7,601,026$ & 76.0 & 0.91 & $4,019,648$ & $6,449,217$ & 64.5 \\
\hline $45-64$ & 0.142 & 94,996 & 13,486 & $1,765,060$ & $3,195,834$ & 33.6 & 0.82 & $1,445,507$ & $2,429,570$ & 25.6 \\
\hline $65-74$ & 0.225 & 81,510 & 18,348 & 723,360 & $1,430,774$ & 17.6 & 0.75 & 543,005 & 984,063 & 12.1 \\
\hline $75+$ & 1.000 & 63,162 & 63,162 & 707,414 & 707,414 & 11.2 & 0.62 & 441,058 & 441,058 & 7.0 \\
\hline
\end{tabular}

${ }^{a}$ Average HRQL for persons in age interval.

HRQL, health-related quality of life; QALE, quality-adjusted life expectancy; QALYs, quality-adjusted life years

plete economic analyses and burden-of-disease studies while reducing the cost and improving the specificity of the analysis. Moreover, since documents compiled by the NCHS, the organization that produces both the mortality data and NHIS data, are free of charge and available on the Internet, it may be possible to conduct burden-of-disease studies and cost-effectiveness analyses with minimal overhead.

In this paper, we explore a simple means of calculating QALYs using publicly available NHIS and mortality data available from the NCHS., ${ }^{4,5,7-9}$

\section{Methods \\ Overview}

To illustrate the use of the YHL measure for the calculation of QALYs and QALE, we calculated the burden-of-disease due to stroke. We first calculated quality-adjusted person-years remaining at birth in an abridged life table cohort using the methods forwarded by Erickson et al. ${ }^{4,7,8}$ (Table 1.) This cohort serves as a reference against which marginal QALYs due to stroke may be calculated. We then generated a second abridged life table in which the cohort is subjected to the risk of dying from all diseases but stroke (a "stroke-free" cohort). Finally, we subtracted the quality-adjusted person-years remaining at birth in the reference life table cohort from the quality-adjusted person-years remaining at birth in the strokefree cohort. All calculations were conducted using abridged life tables on Excel 98 for the Macintosh (Microsoft Inc. Redmond, WA).

\section{Reference Cohort}

An abridged life table is constructed using a hypothetical population in which 100,000 people are born each year. It is assumed that all people in this cohort are at risk of dying or becoming ill, but that there is no migration into or out of the population. Because 100,000 people are born into the cohort each year, the number of persons in the cohort is equal to the number of person-years in the cohort. ${ }^{7,8}$ When person-years are adjusted for HRQL, they become QALYs. Table 1 illustrates how a life table is constructed using the YHL measure to determine the number of QALYs in a cohort.

\section{Use of Published Data}

With reference to Table 1 , Column 1 represents the probability of death in a given interval; Column 2, the number of people alive at the beginning of the age interval; and Column 3 , the number of persons dying during the interval. Using data from an abridged life table, Column-3 values for each interval are entered first. ${ }^{7}$ Column-2 values are calculated as $\mathrm{s}_{\mathrm{x}}=\mathrm{s}_{\mathrm{x}-1}-\mathrm{d}_{\mathrm{x}-1}$, where $\mathrm{s}_{\mathrm{x}}$ is the number of survivors at the beginning of age interval $\mathrm{x}, \mathrm{d}_{\mathrm{x}}$ is the number of persons dying in the interval, and $x-1$ denotes the previous interval. Because all life tables begin with 100,000 persons alive at the beginning of the first age interval, the first value of Column 2 is 100,000 . Dividing the total number of deaths in the interval (Column 3) by the number of persons alive at the beginning of the interval (Column 2), yields the probability of death during the interval (Column 1).

Column 4 row values represent the total number of personyears in the interval and are calculated using the formula $n\left(s_{x}-0.5 d_{x}\right)$, where $n$ is the length of the interval. This formula assumes that all deaths occurred at the midpoint of the interval-an assumption that does not hold for the first year of life (because of infant mortality) or the last age interval (because the interval is of indeterminate length).

The number of person-years in the first year of life is calculated as $100,000 \mathrm{f}+(1-\mathrm{f}) \mathrm{s}_{1}$, where $\mathrm{f}$ is the separation factor ${ }^{8}$ and $s_{1}$ is equal to the number of persons surviving to age 1 . The separation factor is equal to the proportion of infant deaths in the base year occurring to infants born the previous year and is available from the $\mathrm{NCHS}^{8}$ and $\mathrm{s}_{1}$ may be obtained directly from an existing life table. ${ }^{7,8}$ The number of person-years in the first age interval in Table 1 is thus $(n-1)\left(\mathrm{s}_{\mathrm{x}}-0.5 \mathrm{~d}_{\mathrm{x}}\right)+\mathrm{s}_{0} \mathrm{f}+(1-\mathrm{f}) \mathrm{s}_{1},{ }^{7}$ where $\mathrm{d}_{\mathrm{x}}$ is the number of deaths occurring in persons aged 1 to 44 . The probability of death for the $\geq 75$ age interval is equal to the reciprocal of the life expectancy at age 75 .

To obtain cumulative person-years (Column 5) the values for Column 4 are summed backwards such that the value for the first age interval is equal to the sum of all values in Column 4. Each row in Column 6 is calculated by dividing the row value of Column 5 by the corresponding row value for Column 2. The value of the first row in Column 6 is equal to the life expectancy at birth of the cohort and serves to validate the spreadsheet (this should be similar to the life expectancy at birth in abridged life tables). The values in Column 7 represent the HRQL score for the average person in the United States in each corresponding age interval and are available from the NCHS. ${ }^{4}$ Column 8 is equal to the product of Column 7 and Column 4. Columns 9 and 10 are derived in a manner analogous to Columns 4 and 5 .

Since the age intervals must be the same across prevalence, mortality, and HRQL data, it is best to generate age intervals 


\begin{tabular}{|c|c|c|c|c|c|c|c|c|c|c|}
\hline $\begin{array}{l}\text { Age } \\
\text { interval }\end{array}$ & $\begin{array}{l}\quad 1 \\
\text { Probability } \\
\text { of death in } \\
\text { interval }\end{array}$ & \begin{tabular}{l}
\multicolumn{1}{c}{2} \\
Number \\
dying in \\
interval
\end{tabular} & $\begin{array}{l}\text { Person-years } \\
\text { in interval }\end{array}$ & $\begin{array}{l}\text { Life } \\
\text { expectancy }\end{array}$ & Prevalence & $\begin{array}{c}6 \\
\text { YHL } \\
\text { Score }\end{array}$ & HRQL & QALYs & \begin{tabular}{l}
\multicolumn{1}{c}{9} \\
Cumulative \\
QALYs
\end{tabular} & QALE \\
\hline$<45$ & 0.050 & 5,002 & $4,405,283$ & 76.0 & 0.0017 & 0.62 & 0.913 & $4,024,338$ & $6,510,545$ & 65.11 \\
\hline $45-64$ & 0.142 & 13,460 & $1,765,369$ & 33.7 & 0.0149 & 0.47 & 0.825 & $1,458,123$ & $2,486,208$ & 26.17 \\
\hline $65-74$ & 0.224 & 18,238 & 724,195 & 17.6 & 0.0519 & 0.43 & 0.772 & 559,793 & $1,028,085$ & 12.61 \\
\hline $75+$ & 1.000 & 63,300 & 708,964 & 11.2 & 0.988 & 0.38 & 0.660 & 468,291 & 468,291 & 7.40 \\
\hline
\end{tabular}

${ }^{a}$ HRQL score associated with the YHL measure.

HRQL, health-related quality of life; QALE, quality-adjusted life expectancy; QALYs, quality-adjusted life years; YHL, years of healthy life

common to all available data. When using estimates of disease prevalence published by the NCHS, it is easiest to use the four intervals presented in Table 1 (although the use of electronic NHIS data obviates this requirement). Alternatively, the 0 to 45 year interval may be broken into two separate intervals, 0 to 15 and 15 to 44 , producing a total of five age intervals. In doing so, the researcher assumes that the disease prevalence ratio for persons aged 15 to 44 (the interval used in mortality publications) is equal to the prevalence ratio for persons aged 18 to 44 (the interval used to report prevalence ratios).

\section{Use of Electronic Data}

Although the use of electronic data substantially increases the cost and time requirements of cost-effectiveness or burdenof-disease studies, it is possible to use much smaller age intervals, and thus improve the accuracy of model outputs. To generate reliable prevalence and HRQL estimates using electronic data, it is necessary to aggregate 3 to 4 years of NHIS data.

The first row value for Column $1\left(q_{0}\right)$ is approximated using the infant mortality rate, which is equal to the number of deaths in the first year of life divided by the number of live births. Because some deaths occur in infants born the previous year and the number of live births varies slightly from year to year, a more accurate estimate may be obtained using the separation factor, $\mathrm{f}$. The more accurate estimator of $\mathrm{q}_{0}$ is $\mathrm{D}_{0}(1-\mathrm{f}) / \mathrm{B}_{\mathrm{x}}+\mathrm{D}_{0} \mathrm{f} / \mathrm{B}_{\mathrm{x}-1}$, where $\mathrm{D}_{0}$ is the total number of infant deaths and $B_{x}$ is the total number of births in year $x$.

The remaining values for Column 1 are generated using the formula $\mathrm{q}_{\mathrm{x}}=\mathrm{D}_{\mathrm{x}} /\left(\mathrm{P}_{\mathrm{x}}+0.5 \mathrm{D}_{\mathrm{x}}\right)$ where $\mathrm{q}_{\mathrm{x}}$ is the probability of death during the interval, $\mathrm{D}_{\mathrm{x}}$ is the total number of deaths observed at age $\mathrm{x}$, and $\mathrm{P}_{\mathrm{x}}$ is the midyear population for persons aged $x .{ }^{8}$ The survivors at age $\mathrm{x}\left(\mathrm{s}_{\mathrm{x}}\right)$ may then be calculated as $s_{x-1}\left(1-q_{x-1}\right)$. Column 3 values are simply the product of Columns 1 and 2 values for the corresponding rows.

All other values are calculated in an identical manner to those derived from a published life table, with the exception of total person-years in the final age interval. This is equal to the number living at the beginning of the interval divided by the probability of death during the interval $\left(\mathrm{s}_{\mathrm{x}} / \mathrm{q}_{\mathrm{x}}\right)$.

\section{Stroke-Free Cohort}

Table 2 represents an abridged life table for all persons except those with cerebrovascular disease listed as an underlying cause of death (International Classification for Disease, 9th Revision codes 430 to 438 ). Table 2 is calculated in the same manner as Table 1 with a few exceptions. In Column 1, the probability of death in the interval due to the disease under study (in this case, stroke) is subtracted from the all-cause mortality rate.

Column 5 contains age-specific prevalence rates for the disease under study. ${ }^{9}$ These are multiplied by ( $\left.1-\mathrm{HRQL}\right)$ for the disease in question (in this case, the age-specific YHL score for stroke $)^{5}$ in Column 6 . The product of Columns 5 and 6 for each age interval is then added to the average HRQL (Column 7). This is mathematically equivalent to averaging out the HRQL lost to stroke in the cohort. The remainder of Table 2 is calculated in a manner analogous to Table 1.

Finally, we calculated the burden of disease due to stroke by subtracting QALYs remaining in the cohort with all diseases (see Table 1, Column 9, Row 1) from QALYs remaining in the cohort with all diseases except stroke (see Table 2, Column 9, Row 1). To obtain the QALE lost to stroke, the QALE in the reference cohort (see Table 1, Column 10, Row 1) is subtracted from the QALE in the stroke-free cohort (see Table 2, Column 10, Row 1). We validated the spreadsheet model by comparing our QALE value with published reports.

\section{Sensitivity Analysis}

Of the two data sets used to calculate QALYs, only the NHIS data are subject to random error. Since the NHIS is used to generate both HRQL scores and disease prevalence rates, it is possible to calculate the standard error for these parameters. ${ }^{9}$ A discussion of random and nonrandom errors may obtained from the NCHS. ${ }^{9}$

Although it is possible to estimate the standard error of HRQL scores obtained from the YHL measure, the standard error provides no information about the degree to which the scores represent the preferences of particular subgroups for the conditions specified. We have noted previously that the scores are developed from a two-domain model, which necessarily represents a relatively coarse description of a health state. In addition, scores are calculated rather than measured. Finally, the structure of the NHIS does not allow comorbidities to be broken out separately. ${ }^{5}$ For all of these reasons, investigators may wish to conduct sensitivity analyses for the HRQL values by using 25th and 75th percentile values for the conditions studied. These values have been previously published and are available in tabular form. ${ }^{5}$ The extent to which death certificate data are subject to misclassification bias or other types of nonrandom error may be estimated from the 


\begin{tabular}{|c|c|c|c|c|}
\hline & $\begin{array}{l}\text { U.S. population } \\
\text { cohort }\end{array}$ & $\begin{array}{l}\text { Stroke-free } \\
\text { cohort }\end{array}$ & Difference & 95\% CI** \\
\hline Life expectancy & 76.01 & 76.04 & 0.03 & - \\
\hline QALE & 64.49 & 65.11 & $0.61 *$ & $0.60-0.61$ \\
\hline QALY & $6,449,217$ & $6,510,545$ & 61,328 & $60,272-62,383$ \\
\hline
\end{tabular}

*Difference is not exact due to rounding.

**Based on sampling error in prevalence estimate alone.

QALE, quality-adjusted life expectancy; QALY, quality-adjusted life years.

medical literature ${ }^{10-12}$ and sensitivity analyses conducted, as appropriate.

\section{Results}

Life expectancy, QALE, and QALYs remaining at birth for the general U.S. population cohort and the strokefree cohort are presented in Table 3. The burden of disease due to stroke was approximately 61,328 QALYs. Assuming that a negligible number of deaths due to stroke would be attributed to other causes and that the years of healthy life measure produces HRQL scores that are representative of the condition-specific values of the general U.S. population, the 95\% confidence interval for the burden of disease due to stroke in our life table cohort would be approximately 60,272 QALYs to 62,383 age-adjusted QALYs. If stroke were eliminated, persons in the United States would gain approximately 0.61 years of QALE but only approximately 0.03 years of additional life.

The reference cohort produced a QALE at birth of 64.5 years-0.5 years longer than the 1990 NCHS estimate of QALE, 64 years. ${ }^{4}$ Our estimate of life expectancy in 1997, 76 years, differed from the NCHS estimate, 76.5 years, by approximately seven tenths a percent-a variation that is likely attributable to the large age intervals used in this study.

\section{Conclusions}

In this paper, we illustrated a simple technique for calculating QALYs using data accessible by the Internet. To illustrate this method, we calculated QALYs lost to stroke in our cohort, an estimate affected by random error. The model predicted the loss of approximately 61,328 QALYs to stroke in the hypothetical life-table cohort. On average, persons residing in the United States could expect to live approximately 0.61 additional years of quality-adjusted life if strokes were eliminated, but only approximately 0.03 additional years of life when quality adjustments are not included. The difference between absolute and quality-adjusted years gained underscores the importance of including a valuation of quality years lost to morbidity. This method not only allows for rapid tabulation of the burden of disease due to most illnesses, but it also provides a convenient way of calculating the number of QALYs gained due to an intervention in cost-effectiveness analyses.

For researchers wishing to conduct a cost-effectiveness analysis, the differential in QALE for a group with and without a condition will usually provide sufficient data for the construction of a decision analysis model. ${ }^{1}$ Moreover, most interventions will not require de novo generation of HRQL scores using data from the NHIS since these data are readily available in the medical literature. ${ }^{4,5}$

Calculations for the YHL measure are based on the technique of correspondence analysis ${ }^{13}$ which is linked to values drawn from the Health Utilities Index (HUI), ${ }^{14}$ a relatively complex, multi-attribute, health status instrument. Multi-attribute models typically utilize multiple health domains. Given its two-domain structure (role function and self-reported health), HRQL scores derived from the YHL measure may be less precise than scores generated from measures that have a more complex formulation of health such as the HUI, the Quality of Well-Being Index,${ }^{15}$ and the EuroQol. ${ }^{16}$ On the other hand, the measure's ability to include the average HRQL of a population and to generate HRQL scores specific to a demographically defined group may provide advantages over other HRQL indexes in particular settings. Ideally, future population surveys would contain more complex preference-based instruments that could be coupled to specific disease prevalence data.

The NHIS is an annual survey conducted by the NCHS. ${ }^{17}$ Data are collected in face-to-face interviews in a nationally representative sample of households. When conducting our sensitivity analysis, we only included sources of random error in estimating the $95 \%$ confidence interval for the burden of disease due to stroke. There is debate surrounding the sensitivity and specificity of self-report data which are subject to recall bias. ${ }^{18,19}$ A subject's inability to accurately remember the illnesses from which he or she suffers may affect both the tabulation of prevalence rates and the HRQL score. Moreover, the use of death certificates to ascertain mortality rates is subject to misclassification bias. ${ }^{10-12}$ Death certificates may fail to correctly classify 
the primary or underlying cause of death or the demographic characteristics of the decedents.

Because the sample size of the NHIS is small (approximately 103,000 people in 1997),${ }^{15}$ relative to the total number of possible diseases subjects might be afflicted with, the researcher often faces a trade-off between unreasonably large age intervals or too few subjects to generate reliable estimates of prevalence or HRQL. Although African Americans and Hispanics are oversampled in the NHIS, researchers interested in obtaining prevalence rates for other races/ethnicities or for specific geographic regions will not likely be able to generate reliable estimates using a single year of NHIS data. Sample size issues may be overcome by aggregating NHIS data over a number of years. ${ }^{5}$ However, year-to-year changes in disease prevalence may limit this method. Finally, the age intervals we used were broad. When infant mortality is accounted for separately, however, the use of the broad age intervals we present here only produces a slight underestimate of life expectancy (data not shown).

The Panel on Cost-Effectiveness in Health and Medicine $^{1}$ recommends that cost-effectiveness analyses designed to inform resource allocation decisions should present outcomes in the form of QALYs. The method we present for calculating QALYs is simple enough to permit their use in resource-limited research efforts. Using the techniques discussed in this paper, it is possible for public health researchers to generate burden-of-disease estimates specific to demographically defined communities within the span of a few hours. This method may, therefore, also have great utility in assisting public health agencies in making rapid decisions surrounding the allocation of resources to local health priorities.

We are indebted to Marianne Fahs, Clyde Schecter, Peter Franks, and Karla Hansen for their assistance with verifying the statistical accuracy of the calculations in this paper.

\section{References}

1. Gold MR, Siegel JE, Russell LB, Weinstein MC, eds. Cost-effectiveness in health and medicine. New York: Oxford University Press, 1996.

2. Cox DR, Fitzpatrick R, Fletcher AE, Gore SM, Spiegelhalter DJ, Jones DR. Quality of life assessment: can we keep it simple? J R Statist Soc 1992;35393.

3. Hoyart DL, Kochanek KD, Murphy SL. Deaths: final data for 1997. National Vital Statistics Report 1999;47:1-146.

4. Erickson P, Wilson R, Shannon I. Years of healthy life. Statistical Notes 1995; 7:1-14.

5. Gold MR, Franks P, McCoy KI, Fryback DG. Toward consistency in cost-utility analyses: using national measures to create condition-specific values. Medical Care 1998;36:778-92

6. Centers for Disease Control and Prevention. Health measures in the new 1997 redesigned National Health Interview Survey (NHIS). Available at: www.cdc.gov/nchs/about/major/nhis/hisdesgn.htm. Accessed Jan 18, 2000.

7. Anderson RN. Life tables, 1996. National Vital Statistics Report 1998;47:120.

8. Anderson RN. Method for constructing complete annual U.S. life tables. Vital Health Stat 2 1999;129:1-36.

9. Benson V, Marano MA. Current estimates from the National Health Interview Survey, 1995. Vital Health Stat 1998;10:1-105.

10. Rosamond WD, Tyroler HA, Chambless LE, Folsom AR, Cooper L, Conwill D. Educational achievement recorded on certificates of death compared with self-report. Epidemiology 1997;8:202-4.

11. Kelly JJ, Chu SY, Diaz T, Leary LS, Buehler JW. Race/ethnicity misclassification of persons reported with AIDS: the AIDS Mortality Project Group and the Supplement to HIV/AIDS Surveillance Project Group. Ethn Health 1996;1:87-94.

12. Rosenberg HM, Maurer JD, Sorlie PD, et al. Quality of death rates by race and Hispanic origin: a summary of current research, 1999. Vital Health Stat 2 1999;128:1-13.

13. Greenacre MJ. Correspondence analysis in practice. London, UK: Academic Press, 1993.

14. Feeney DH, Furlong W, Barr RD, Torrance GW. Multiattribute health status classification systems: health utilities index. PharmacoEconomics 1995;7:490-502

15. Kaplan RM, Anderson JP. A general health policy model: update and applications. Health Serv Res 1988;23:203.

16. The EuroQol Group. EuroQol: A new facility for the measurment of health-related quality of life. Health Policy 1990;16:199.

17. Centers for Disease Control and Prevention, National Center for Health Statistics. NHIS survey description, 1997. Available at: www.cdc.gov/nchs/ nhis.htm. Accessed March 10, 2000.

18. Robinson JR, Young TK, Roos LL, Gelskey DE. Estimating the burden of disease: comparing administrative data and self-reports. Med Care 1997; 35:932-47.

19. Edwards WS, Winn DM, Kuriantzick V, et al. Evaluation of National Health Interview Survey diagnostic reporting. Vital Health Stat 1994;2:1. 